\title{
2015 WSGC Elijah High-Altitude Balloon Payload Project Final Report
}

\section{September 14, 2015}

\author{
Ben Jensen ${ }^{1}$, Nate Klassen ${ }^{2}$, Woodrow Walker ${ }^{1}$, Scott Frazier ${ }^{3}$, Kai Swanson ${ }^{1}$, Taylor Davitz ${ }^{1}$ \\ ${ }^{1}$ Milwaukee School of Engineering, ${ }^{2}$ University of Wisconsin-Whitewater, ${ }^{3}$ University of Wisconsin- \\ Milwaukee
}

\begin{abstract}
The purpose of this report is to discuss the research, development, and findings of several experiments performed on a high-altitude balloon payload platform. The team decided of the following five experiments to be performed during the flight: Oxygen Generation, Internal Heating, Speed of Sound Properties, Ozone Levels, and a new payload structure concept. The entire team was required to research and develop new skills to effectively design our experiments, including: Arduino programming, 3-D modeling/printing, soldering, launch equipment handling, and construction techniques. While the launch was successful, we did fall short of our overall goal in terms of data acquisition. The internship proved to be a rewarding introductory experience to real-world engineering. The project help us learn how to implement the entirety of the engineering process, using each person's strengths to achieve an objective.
\end{abstract}

\section{Introduction}

The goal for the internship team was to research, design, and execute experiments aboard a highaltitude balloon platform. The team would collect and analyze data from each experiment. There were several constraints for the project, including budget, weight, and size. The team was given full control over what we wanted to research, and decided five upon concepts: Oxygen Generation, Internal Heating, Speed of Sound Properties, Ozone Levels, and a new payload structure concept.

\section{Oxygen Generation}

As the altitude increases, we see a corresponding decrease in air pressure. This creates an increasingly hostile environment for human life, since as the air pressure decreases, there is less breathable air. With this idea in mind, we set out to research methods of producing breathable air in the near-space environment.

Purpose: There are several methods for producing oxygen that we could have used in this experiment. We narrowed it down to three options, from which one would be chosen for the experiment. These options were: electrolysis, ozone-UV manipulation, and perchlorate candles. The ozone-UV manipulation required the use of a heavy and expensive power source and the perchlorate candles required an open ignition source, which was not allowed aboard the payload. That left us with the electrolysis method, which we decided could be feasible after preliminary research.

We would like to thank WSGC and Dr. William Farrow for making this project possible 
Method: The method for producing oxygen from an electrolytic solution is demonstrated in a device called a Hoffman Voltameter. The idea is to use the solution to conduct a current, which separates the oxygen and hydrogen of the water at each electrode. This allows us to produce both gaseous oxygen and hydrogen from the electrolytic solution

However the Hoffman Voltameter is not appropriately constructed to be used on a high-altitude balloon platform, so we would have to redesign it. We decided on a 5\% Sulfuric acid solution as an electrolyte, as this would be much more conductive than water or saltwater. We determined that the weak concentration would not be a problem when interacting with other components, though we would eventually be proven wrong in this regard.

We used PVC tubing and polyurethane tanks to house the liquid, with a small PVC tube connecting the two tanks to form the bridge for

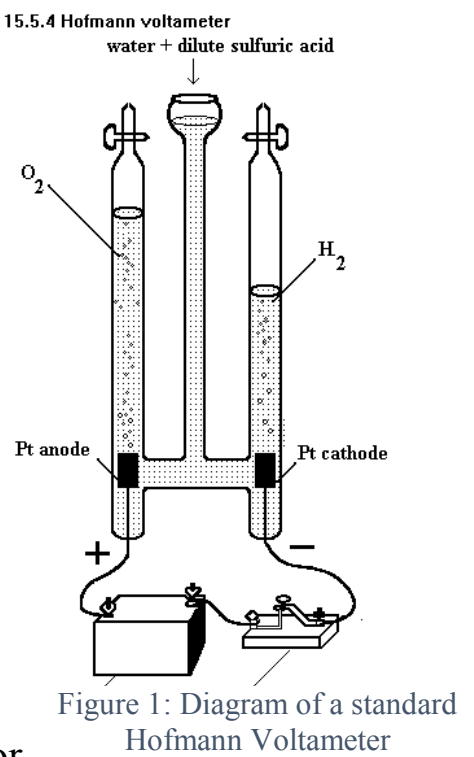
the electric current. Early tests with the acid showed that the rate of gas production would be much more than we had previously thought. We resolved this by using a cylindrical electrode in place of copper plates.

Another issue that would have to be addressed is the buildup of gas pressure as the gas is produced in a fixedvolume container. To combat this, the team had to design an adjustable-volume system for the displaced solution to flow into, thus keeping the internal gas pressure at about fifteen psi. The displaced fluid would flow into a large syringe connected by tubing. A small LMP pressure sensor was connected to the fluid system, which would monitor internal fluid pressure, which would increase with gas pressure. This pressure sensor would control a Firgelli

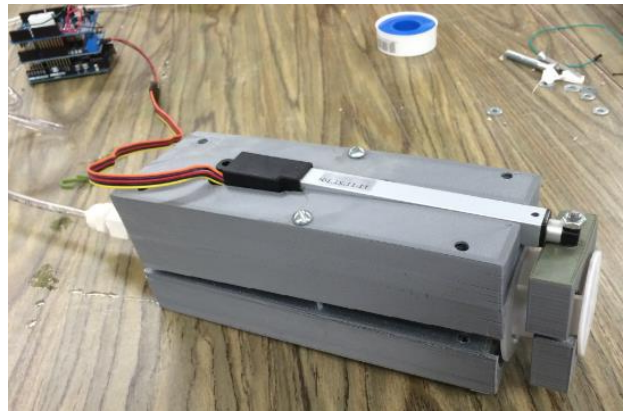

Figure 2: Image of the actuator-syringe system linear actuator. The actuator moved the plunger on the syringe, thus adjusting the volume of the entire electrolytic system to keep it at fifteen psi. In order to maintain a straight alignment while the syringe was being moved, a chassis for the syringe was 3-D printed. An image for the volume-control system can be seen in Figure 2. In the event that the syringe became full, the current through the solution would be cut off using an integrated relay, ceasing all gas production.

Results: We knew that this experiment had a decent chance of failure, which we were willing to accept. As such, the experiment was not in any condition to fly. The volume manipulation was successful, though all of the extra hardware needed to ensure success created a weight problem, which, when combined with the large weight of the solution already present, proved to be insurmountable. Another problem arose with the solution itself, as the 2-part resin/hardener that we used in the final sealing process ended up reacting negatively with the acid solution, causing the hardener to fail. These setbacks occurring so late in the design process did not leave us with enough time to redesign the system to rectify the problems with weight and leakage. 
Conclusion: This component of the balloon design was the most complicated experiment we wished to perform aboard the payload. We set out knowing that there was a chance that the experiment would not fly. There are several changes that I would make to the design for future attempts. Even though the conductivity is low, using a less volatile electrolyte would have been much easier, especially in the testing stage. The flexible tubes should be replaced with rigid, solid pipe connections, which are easier to seal and maintain. The tanks should have been smaller to reduce weight, which would have let us create a smaller volume-control system, saving more weight. Finally, a heavier duty sealant should have been used. We tried using JB Weld in early design stages, but the tubing proved to be incompatible with JB Weld's long drying time. With rigid pipe connectors, this problem could have been addressed more effectively. Overall, I believe that we laid important groundwork for what could be an interesting experiment for future payload teams.

\section{Internal Heating}

The team began with experimental brainstorming. In this process the temperature of the payload affected several the experiments that were possible. For example, the $\mathrm{CO}_{2}$, Ozone, and sound sensors were constrained because they were rated to work at a certain temperature. (In most cases the limit was -40 degrees Celsius.) It was found that the temperatures on the inside of the payload would reach minus 20 degrees Celsius and the temperatures reached at around 100,000 feet were about minus 60 degrees Celsius.

\section{(http://www.aerospaceweb.org/question/atmosphere/q0112.shtml)}

Even though the payload would have insulation, there would be a change in temperature of eighty degrees Celsius at least.

Purpose: Our goal with this experiment was to maintain an internal temperature of 0 degrees Celsius. In order to accomplish this goal we brainstormed and researched several ideas. However, the one that seemed the most practical was to utilize the large change in temperature via a Peltier Junction. If one was used, power would be able to be generated and hopefully turned into heat to maintain the temperature of our payload.

Method: First the cell would have to gather heat from the inside, where it is pumped to a heat exchanger on the outside of the box. The collected heat is then released to the ambient air to warm the payload. This would all be accomplished using two heat sinks and fans in conjunction with a Peltier device.

The smaller heat sink was coded to temperature below that of the air in the box. The sink would then pick up the heat as the air circulates between the fins. The Peltier device is mounted

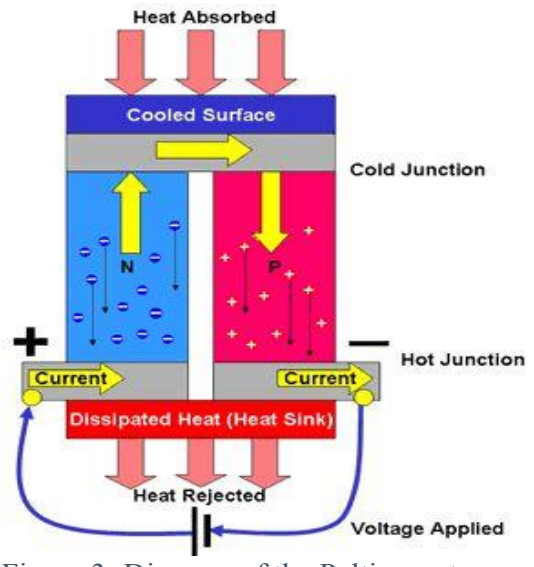

Figure 3: Diagram of the Peltier system between the low-heat sink and the larger high-heat sink. A fan located on the hot side would circulate ambient air between the sink's fins to absorb the collected heat. This would include the heat from the box and the heat produced by the cell itself. The outline of this process is demonstrated in Figure 3. 
By using the device in Figure 3 with a reversed polarity, we could create a heater. After researching Peltier Cells for the purpose of creating thermal energy, it was found that power generation was not feasible. The change in temperature between the cold and hot nodes must be greater than 100 degrees Celsius for any significant energy to be harnessed especially since our power supply was restricted to any battery $9 \mathrm{~V}$ and less due to the six pound weight restriction. In addition, the Peltier Cell requires $12 \mathrm{~V}$ or higher for an effective heating device. As a result, we decided to investigate a resistive heater as a means for heating the inside of the payload.

Even though the Peltier Cell was not feasible we could still use a simpler method of heating with energy generated though resistivity. Similar to the Peltier system, power can be generated through equation (1) below. The rate of heat transfer, q, is proportional to the power generated multiplied by time. Therefore if we have more current then the payload should get hotter over a shorter period of time [see equation (2)]. However, the current is restricted greatly by the voltage source and, in this case, we are limited to a $9 \mathrm{~V}$ battery due to weight.

$$
\begin{gathered}
\mathrm{P}=\mathrm{i}^{2 *} \mathrm{R}(1) \\
\mathrm{q} \propto \mathrm{i}^{2} * \mathrm{R} * \mathrm{t}(2)
\end{gathered}
$$

In researching the heater, we found a $10 \times 5 \mathrm{~cm}$ metal-polymer fiber composite conductive yarn sold through Adafruit Industries. To find the power that it drains we took a multimeter to find its resistance. Using equation (1) and a $9 \mathrm{~V}$ battery as its energy source the power was found to be 1.05 Watts. With the feasibility of the heater and our power source we decided to go with this configuration because it was the best that we could do in the given flight conditions and constraints.

The following was used in the fabrication of the heater:

- Arduino Mega

- MCP9809 digital temperature sensor

- N Channel MOSFET

- Lithium Ion 9 V Battery

The Mega was chosen so that multiple devices could be used along with the SD shield on the controller. The temperature sensor has a typical accuracy of $\pm 0.25^{\circ} \mathrm{C}$ over the sensor's $-40^{\circ} \mathrm{C}$ to $+125^{\circ} \mathrm{C}$ range and a precision of $+0.0625^{\circ} \mathrm{C}$ according to the Adafruit Ind. datasheet. The MOSFET was chosen so that code could be written to make the device act like a thermostat. . If the temperature is below 25 degrees then it sends a signal to the MOSFET to turn on the heater. If temperature is at the designated temperature of 25 then the code will emit "OFF" and the heater will turn off. One advantage of using the polymer fabric we have is that once the current is shut off from the heater, it cools very quickly. This allowed us to qualitatively feel if the MOSFET was working during testing. Lastly, a lithium ion battery was chosen because the device needed to last about three hours. The battery allows us to do so because of the higher rated current. 
Results: We were not able to have the initial design that was wanted however the heater did increase the temperature on the inside of the payload, and the MOSFET allowed us to control the temperature to the highest extent we could with our design. The temperature in Figure 4 does indeed drop to minus 20 degrees Celsius as expected with the high altitude, but the temperature begins to increase shortly after. The heater was limited to a certain wattage level therefore it can only increase temperature slowly. In future studies, we would like to look into pulse width modulation for a more accurate

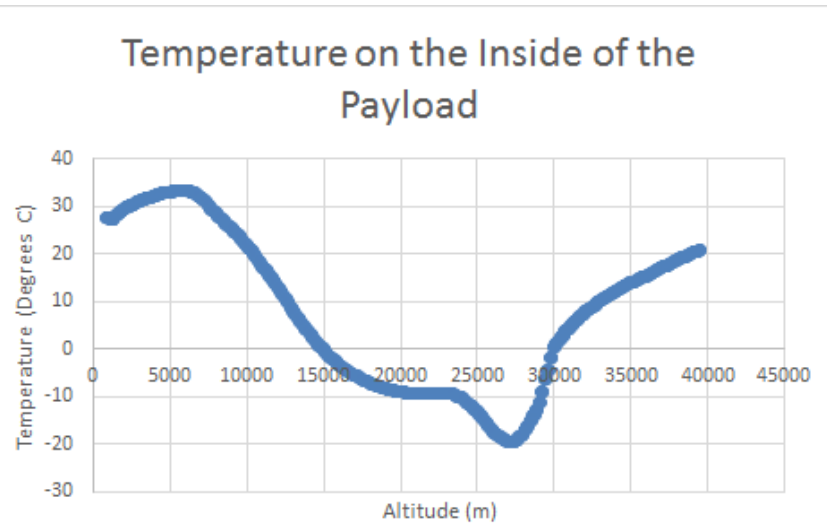

Figure 4: Graph of internal temperature over altitude during the flight temperature control along with a larger power source.

Conclusion: The internal heating system worked better than expected during the flight, though the results show it to be relatively inconsistent, where we had large drops in temperature at one point in the flight, however the temperature eventually rose in the higher stages of the flight, with a $20^{\circ} \mathrm{C}$ internal temperature at 40,000 meters. We believe that the experiment was a success.

\section{Speed of Sound Properties}

One of the interesting results of being in a low pressure environment is the change to how we hear the world. Without a substantial medium to travel through, the properties of sound start to change, primarily the speed of sound, which is around $340 \mathrm{~m} / \mathrm{s}$ at sea level.

Purpose: This experiment was designed to test how the speed of sound changes as the balloon goes higher in the atmosphere. The speed of sound is dependent on two main variables; temperature, and the composition of the medium it is traveling through Figure 4. As the payload rises the speed of sound should change due to the change in the medium, temperature will be negligible because the whole test will be in a heated chamber. We hope to see a linear relationship such as the one in Figure 4.

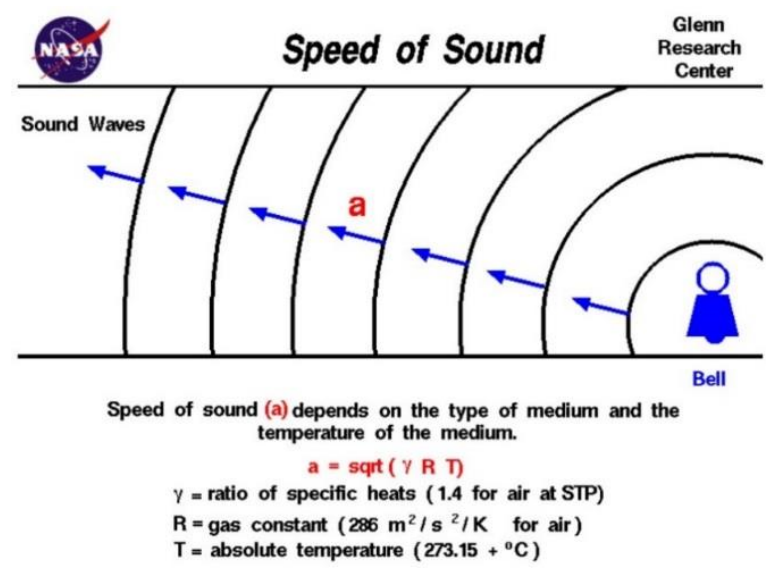

Figure 5: Diagram of how speed of sound changes with heat

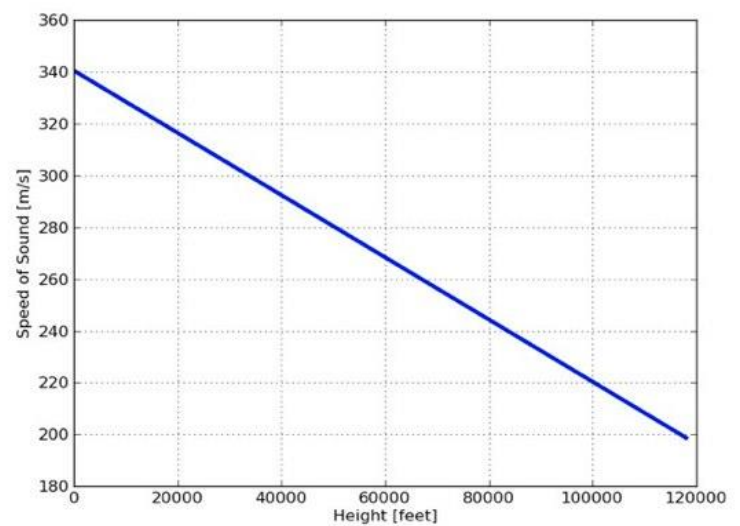

Figure 6: Graph of speed of sound as it changes with height 
Method: As an initial idea the team came up with the simple idea of having a speaker and a microphone set a certain distance apart. A $77 \mathrm{~Hz}$ tone would be played by the speaker, and it would then be timed to see how long it took to get to the microphone using a timer programmed on the Arduino. The initial problem with this idea was that there was too much background noise, even in a container that was designed to keep out ambient sound. The next step to fix this problem was to implement a band-pass filter between the microphone output and the Arduino. This filter would help filter out frequencies that are not in the same range as the $77 \mathrm{~Hz}$ signal. A simple high pass and low pass filter was created using a $2.1 \mu \mathrm{F}$ and a $2 \mu \mathrm{F}$ capacitor and two $100 \mathrm{ohm}$ resistors.

With the addition of the band-pass filter the readings were better, but there was still too much background noise to get an accurate reading. When the program was run for ten seconds with the tone playing continuously and data points being taken every eighth of a second, the Arduino was only able to recognize the tone about $15 \%$ of the time. With some research it was decided that a 741 op amp band-pass filter would be more accurate. When the filter was tested the same way as the original filter the results were better than before, with a percentage of $21 \%$, but still not accurate enough.

At this point some extra testing was done and the problem was discovered. With a constant input of analog values into the Arduino, the analog ports themselves were adding feedback. There are methods to help with the Arduino feedback, but we decided to go a different route. We decided to test an ultrasonic module that uses the pulse width modulating (PWM) digital ports on the Arduino. The ultrasonic works by sending out a ping from the trig pin which then bounces off whatever is in front of it. It then comes back and triggers the echo pin sending a pulse to the Arduino board. Then using the PWM, which measures the length of the pulse, the length of the pulse in microseconds is the time it took for the ping to traverse the distance and bounce back. The speed of sound could then be calculated by taking the distance between the sensor and the object the ping bounced off of multiplied by two, as the ping went that distance twice so we had to account for that, and the time in microseconds. A test was done to determine how accurate the sensor was, and we found that the sensor gave us a reading that was accurate with a $2-3 \%$ in error.

Next was the housing for the experiment. We decided on a two inch diameter rocket tube body. The sensor is mounted on one end with the other end being closed off with painter's tape. The whole tube was 3.25 inches long. This housing would give the experiment a safe environment to test in, without being moved during flight. See Figure 7 for a visual of the housing.

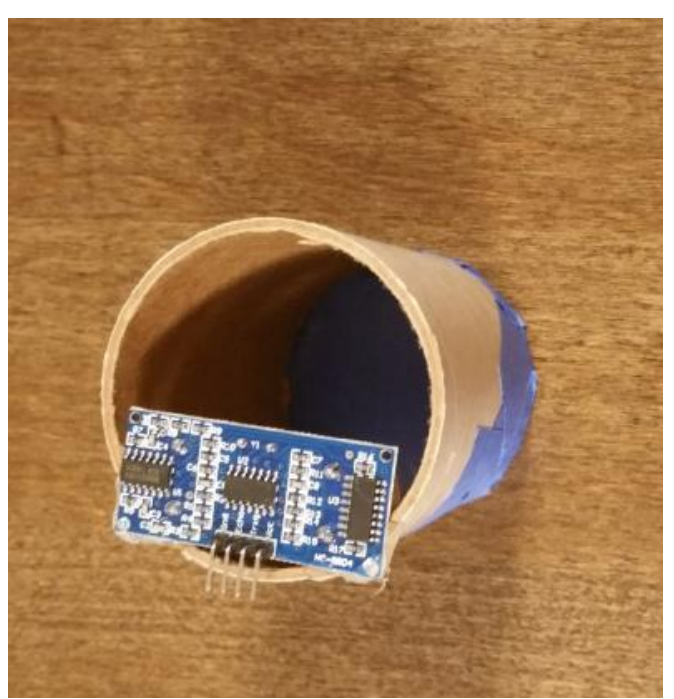

Figure 7: Image of the rocket tube housing with speaker attached 
Results: The expected results from this experiment was that the speed of sound would slow as the balloon rises in the atmosphere. The Arduino took a data point every two minutes, and the goal was to get a set of data with the speed of sounding trending towards zero. Unfortunately we were unable to get a data set from this experiment because during launch a wire was disconnected which caused the sensor to send incorrect readings during the entirety of the flight.

Conclusion: The unfortunate equipment malfunction prevented us from retrieving our results. While the experimental systems worked well during the ground tests, and we believe that they would have worked during the flight, we obviously should have devoted more time to ensuring that the hardware could survive the flight. We could have built in redundancy systems to the circuits that could have bypassed the broken solder and allowed the system to function.

\section{Ozone Detection}

Climate Change and pollution are urgent issues that face us today, and keeping tabs on the Carbon Dioxide and Ozone in the atmosphere is one way to monitor our environmental impact. A cheap and effective way of doing this is to send a simple set of sensors connected to an Arduino with the payload. The parts included the MQ131 Ozone sensor, the MG811 Carbon Dioxide Sensor, and an Arduino Uno. Both of the sensors are built physically identical, but they use different chemical reactants to detect different gasses. See Figure 8 for more technical details on the two sensors. Unfortunately, due to technical limitations, we had to cut the $\mathrm{CO} 2$ experiment entirely.

Purpose: The overarching goal was to take sensor data from the flight, model it against predictions made by previous similar surveys, and report the difference. First, in order to achieve this goal, the sensors must be calibrated. Secondly, they must have a way to log their data in real time. Third, they must be capable of taking accurate readings at high altitude.

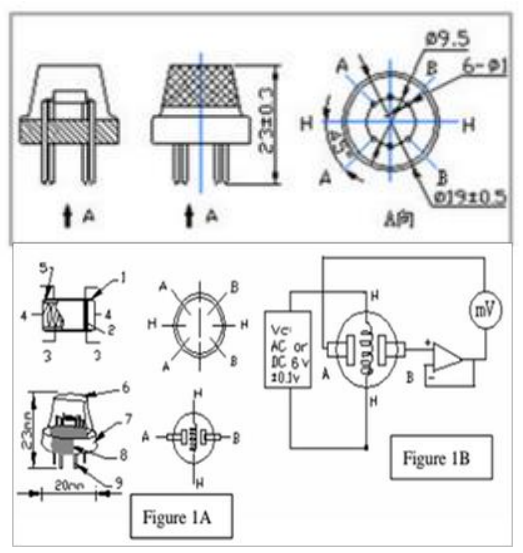

Figure 8: Diagram of sensors used within the ozone experiment

Method: The first experiment was to ensure that the sensors were both operable. Unfortunately, the $\mathrm{CO}_{2}$ sensor failed this first test. Using the $\mathrm{CO}_{2}$ sensor manufacturer's program code, it produced results that were completely illegible. The ozone sensor, on the other hand, produced quantifiable results. In order to ensure the efficacy of the results, two experiments are required: 1) exposing the ozone circuit to extreme low pressure and checking the response, and 2) calibrating it against an existing and functioning ozone sensor. Unfortunately, we were never able to find a sensor to use for calibration, so it flew un-calibrated. In order to log the data in real time, we needed an SD card Arduino shield, which we provided for the group. The ozone sensor was temperature dependent, as well as other aspects of the payload, so to combat this issue, we provided the heating aspect to this project. 
The physical dimensions of the sensors are inconsequential to the overall space constraints of the payload, so that was not a concern. The parts were mounted and added to the other breadboard already in use by other aspects of the payload experiments, so mounting was of little concern.

Results: We decided to keep the ozone sensor as a part of our final payload, but we cut the $\mathrm{CO}_{2}$ sensor due to difficulties and the limitations of the sensor itself. The idea was that the ozone sensor would give acceptable data given the temperature kept relatively constant, and the sensor performed on launch day. However, the temperature did not remain constant, and due to the sensor being un-calibrated, the data received remains indecipherable without calibration.

Conclusion: We believe that improved functioning with the internal heating system would have allowed us better results with the ozone detection system. As it stands, we were not able to interpret the results of the experiment.

\section{Payload Structure}

Purpose: Previous year's payload structures all seemed to revolve around the use of a capsule to protect the onboard experiments from the environment. Our team wanted to go a bit further than that with our payload structure. We wanted to create a shape that could use the windy nature high altitudes to remain stable.

Method: When creating the payload structure, the concept of using a design that created the least amount of spin was used as the key factor, besides the actual structural properties. Looking at past designs, it was easy to see that most of them were spherical in shape and that is what caused them to spin radically. In order to prevent the spinning, the basis of a flag or sail idea was used to conceptualize the shape. To properly store experiments, this sail had to be widened to create the space needed. This then led to the idea of using a triangular prism design. See Figure 9 for a visualization of our structure.

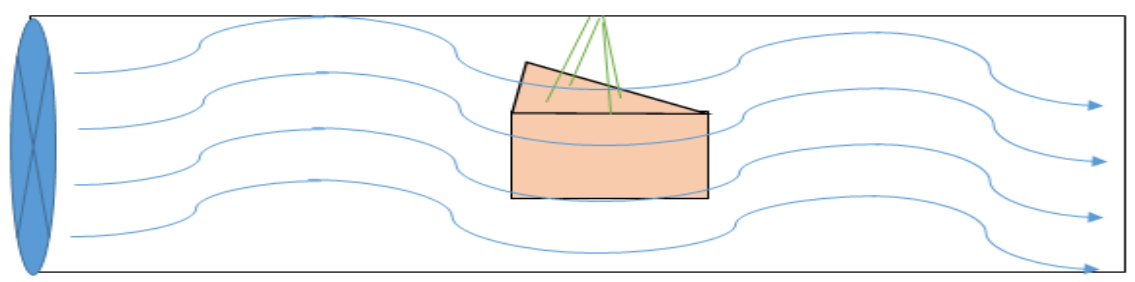

Figure 9: Image of the new structure design. Here it is shown conceptualized during wind tunnel testing 
Once the overall shape was determined, the actual characteristics of this prism had to be determined. Using a wind tunnel, a series of tests in which individual factors of the prism where changed, face width, tail length, face shape, and mounting point shape, it was determined that the best shape to use was a triangular prism with a width to length ratio of $3: 1$, a flat face, and a trapezoidal mounting system to hold it to the balloon. See Figure 10 for details.
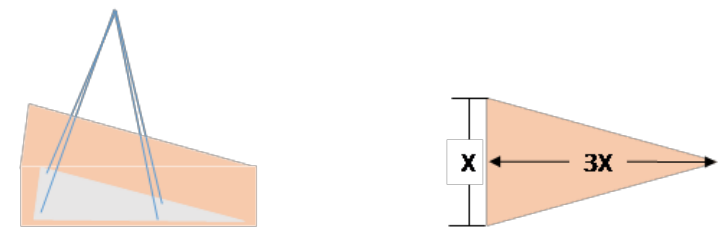

Figure 6: Layout of size proportions and string support locations of the structure

Results: With the addition of the final size specifications of each experiment, the interior support system for the payload could finally be designed and built. Due to the restrictions brought up by the oxygen renewal system, the payload had to be tall, thin, and long. The oxygen system would only take up the exteriors of the payload and leave the top $2 / 3$ 's open. To make the best use of this space and still keep everything in the payload securely attached to the balloon, a base-plate was created on the top and then all the corresponding experiments would hang from it; this was done using an upside down shelves. The drop shelving system worked by using washers and bolts to connect each shelf to four threaded rods that extended the entire length of the payload. These four rods were also used then to connect the top of the payload to the rest of the insulation board sheathing that made up the exterior of the payload.

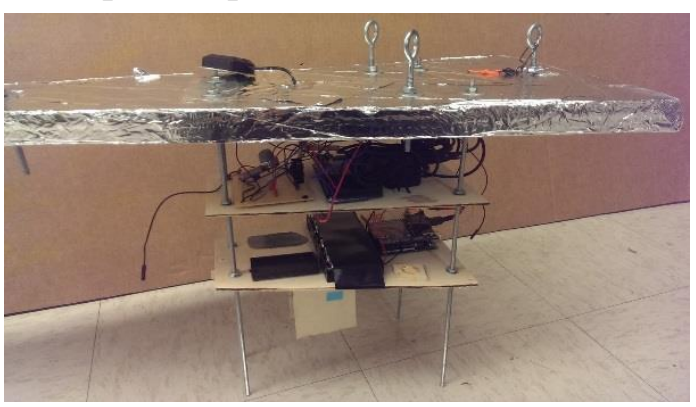

Figure 11: Payload electronics board before the flight

Conclusion: The different payload caplsule worked well for our design, allowing us easy attachment to the balloon, while simultaneously giving us enough room for the moving actuator systems included with the oxygen generation system. While the oxygen generation system was not included in the flight, the structure did a nice job for compensating for the large amount of space required by it. The system also worked nicely for allowing us to run wires from power sources to their respective experimental systems. Overall, the structure was successful in its design. 


\section{Project Conclusion}

Our team had a set of ambitious objectives in mind for this experiment. We knew full well that several of them might lead to failure. Some of the experiments turned out well, while others laid important groundwork for future teams to work with, if they choose to do so. We all learned much about experimental design and the engineering process. Overall, our experience with this project was invaluable in teaching us skills in design, teamwork, communication, and problem-solving. 